\title{
氧鎓离子活化的烯丙基醚原位氧化/脱羧芳构化串联反应:
}

\section{一种合成 $N$-烷基吡咯的新方法}

\author{
李旭涁 ${ }^{a}$ 周 晨 ${ }^{a} \quad$ 刘星粀 $^{a}$ 王 腾 ${ }^{b}$ 虞心红*,a \\ 马红梅*,a 李翠清*,b \\ $\left({ }^{a}\right.$ 华东理工大学药学院制药工程系 制药工程与过程化学教育部工程研究中心 上海市新药设计重点实验室 \\ 生物反应器工程国家重点实验室 上海 200237) \\ ( ${ }^{b}$ 北京石油化工学院 化学工程学院 北京 102617)
}

\begin{abstract}
摘要 发展了一种氧鎓离子活化的烯丙基醚原位氧化/脱羧芳构化串联反应合成 $N$-烷基吡咯化合物的新方法. 该反应 涉及 $\mathrm{C}-\mathrm{N}$ 键的形成和 $\mathrm{C}-\mathrm{O}$ 键的断裂，为制备 $N$-烷基吡咯提供了新途径，收率为 $29 \% \sim 71 \%$, 具有较好的官能团耐受 性.

关键词＼cjkstart梄氧化; 芳构化; 吡咯; 合成方法
\end{abstract}

\section{ipso-Oxidation of Allyl Ether/Decarboxylative Aromatization Cascade Strategy via Oxocarbenium Activation: A Novel Approach for Synthesis of N-Alkyl Pyrroles}

\author{
Li, Xubin ${ }^{a}$ \\ Zhou, Chen ${ }^{a}$ \\ Liu, Xingtong ${ }^{a}$ \\ Wang, Teng ${ }^{b}$ \\ Yu, Xinhong*,a \\ Ma, Hongmei*,a \\ Li, Cuiqing ${ }^{*, b}$ \\ $\left({ }^{a}\right.$ Engineering Research Center of Pharmaceutical Process Chemistry, Ministry of Education, Shanghai Key Laboratory of \\ New Drug Design, State Key Laboratory of Bioengineering Reactors, School of Pharmacy, \\ East China University of Science \& Technology, Shanghai 200237) \\ $\left({ }^{b}\right.$ College of Chemical Engineering, Beijing Institute of Petrochemical Technology, Beijing 102617)
}

\begin{abstract}
An ipso-oxidation of allyl ether/decarboxylative aromatization cascade strategy is reported, resulting in the formation of $\mathrm{N}$-alkyl pyrroles via oxocarbenium activation. This transformation, which involves formation of $\mathrm{C}-\mathrm{N}$ bond and cleavage of $\mathrm{C}-\mathrm{O}$ bond, provides a novel protocol that furnishes $\mathrm{N}$-alkyl pyrroles in $29 \% \sim 71 \%$ yields with good functional group tolerance.

Keywords oxidation of ether; aromatization; pyrroles; synthetic methods
\end{abstract}

\section{Introduction}

Pyrrole moiety is frequently found in natural compounds, pharmaceuticals and biologically active products. Due to their special characterization, pyrroles are also exploited in electrochemistry. ${ }^{[1]}$ Over the past few years, many efforts have been devoted to decarboxylative formation of $N$-alkyl pyrroles. ${ }^{[2]}$ Seidel and coworkers ${ }^{[2]}$ demonstrated a method for the rapid synthesis of $N$-alkyl pyrroles starting from aldehydes or ketones with trans4-hydroxy- $L$-proline in the presence of benzoic acid under microwave irradiation (Scheme 1, a). Subsequently, our group reported an acetic acid-catalyzed efficient access to $N$-alkyl pyrroles, refluxing in $N, N$-dimethylformamide (DMF) in good to excellent yields. ${ }^{[2 e]}$ Additionally, Nageswar and coworkers ${ }^{[2 f]}$ established a protocol using benzyl bromides and trans-4-hydroxy- $L$-proline to synthesize

\footnotetext{
*Corresponding authors. E-mail: xhyu@ecust.edu.cn; hmma@ecust.edu.cn; licuiqing@bipt.edu.cn Received April 13, 2019; revised May 7, 2019; published online May 28, 2019.

Project supported by the National Natural Science Foundation of China (No. 21476078) and the Science and Technology Commission of Shanghai Municipality (No. 12431900902).

国家自然科学基金(No. 21476078)、上海市科学技术委员会(No. 12431900902)资助项目
} 
$N$-substituted pyrroles (Scheme 1, b). However, to the best of our knowledge, the reaction of ethers as initial reactants with $\alpha$-amino acids has never been reported.

$$
\text { Previous work }
$$

This work

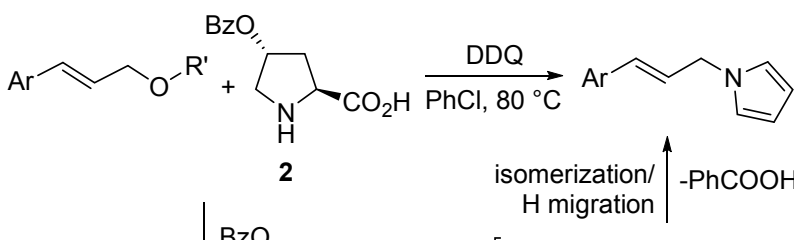<smiles>[R]OC(=O)/C=C\[Al]C</smiles>

Scheme 1 Previous Work and This Work

Ethers occupy an important position in organic chemistry and they are often employed as solvents and in protecting hydroxyl groups owing to their favorable stability. Oxidation of ether could be classified mainly to: (1) oxidation of ether to form carbonyl compounds, ${ }^{[3]}$ and (2) $\alpha-\mathrm{C}_{\mathrm{sp}^{3}}-\mathrm{H}$ cross-dehydrogenative coupling (CDC) reaction of ether. ${ }^{[4]}$ This oxidative CDC reaction of ether goes through oxocarbenium ion intermediates which constitute a class of highly activated species. ${ }^{[5]}$ The reactions of oxocarbenium ions are widely applied in the synthesis of natural products and bioactive molecules. In oxidative CDC reaction, several possible mechanisms of the formation of oxocarbenium ions are illustrated in Scheme 2, including single-electron transfer followed by hydrogen atom transfer and one-step hydride transfer. ${ }^{[6]}$

Herein, it was envisioned that allyl ethers could be oxidized to the corresponding oxocarbenium ions for higher reactivity to enforce decarboxylative aromatization. Gratifyingly, in this study, a novel protocol combined oxidation of allyl ether with decarboxylation has been developed, getting access to $N$-alkyl pyrroles via oxocarbenium activation (Scheme 1). trans-4-Benzoyloxy- $L$-proline 2 was utilized in place of $L$-hydroxyproline, which elimination tends to occur, thus the reaction could proceed under milder condition.

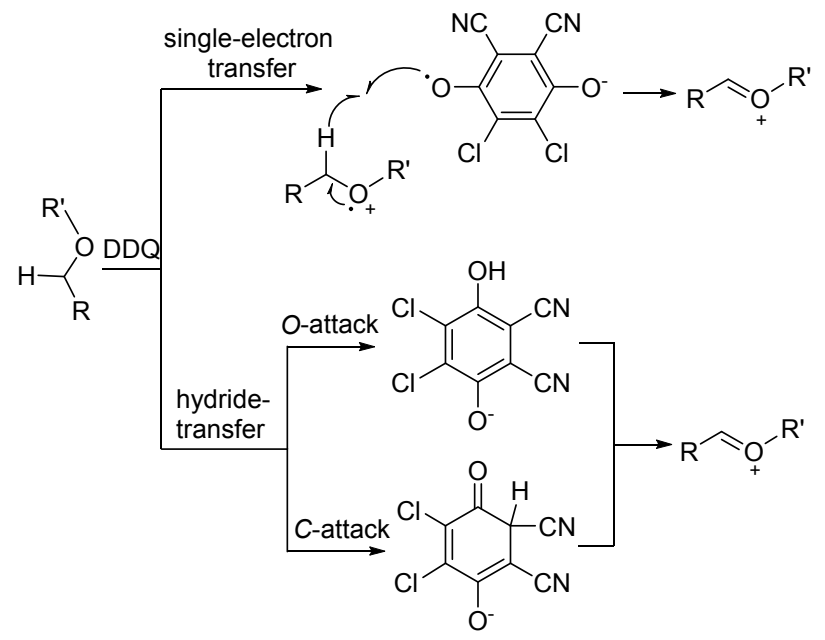

Scheme 2 Formation of oxocarbenium ions from ethers

\section{Results and discussion}

In the initial exploration, it was found that when the reaction was carried out with 2,3-dichloro-5,6-dicyano-1,4benzoquinone (DDQ) (2.0 equiv.) in toluene at $80{ }^{\circ} \mathrm{C}$ for $12 \mathrm{~h}$, the desired product 3a could be detected in $27 \%$ yield based on ${ }^{1} \mathrm{H}$ NMR analysis (Table 1, Entry 1). In addition, the yield decreased when the cinnamyl ether 1 was oxidized before adding $L$-hydroxyproline derivative $\mathbf{2}$ compared with the one-pot condition (Table 1, Entry 2). Further investigation of various solvents revealed that chlorobenzene was the best (Table 1, Entries 4 9). The yield was improved largely in the presence of antioxidant 300 (5-tert-butyl-4-hydroxy-2-methylphenyl sulfide) (1.0 equiv.) (Table 1, Entry 14). The isolated yield of 3a could be further increased to $71 \%$ when the substituent group is ethyl instead of methyl, isopropyl or tert-butyl (Table 1, Entries 16 18).

Under the optimized reaction conditions, a range of allyl ethers were explored. Both the electron-donating and electron-withdrawing groups on the aromatic ring were all tolerated though neither of them performed better than the unsubstituted one (Table 2). Especially when the functional groups were strong electron-donating or electron-withdrawing groups, the yields reduced a lot. In addition to aromatic groups, heteroaromatic groups such as furyl and thienyl were also employed to afford the products $\mathbf{3 1}$ and $3 \mathbf{m}$, albeit in low yields ( $41 \%$ and $29 \%$, respectively). Moreover, indoline-2-carboxylic acid was substituted for $L$-hydroxyproline derivative $\mathbf{2}$ as the reagent, $N$-alkyl indole 30 was obtained.

Based on the above experimental results and previous reports, ${ }^{[2,6]}$ a plausible mechanism is proposed in Scheme 3 . The first step involves oxidation of ether $\mathbf{1}$ to give oxocarbenium ion $\mathbf{A}$, followed by addition of $L$-hydroxyproline derivative $\mathbf{2}$ to $\mathbf{A}$, gaining species $\mathbf{B}$. Subsequent formation of hydrogen bond $(\mathrm{O} \cdots \mathrm{H}-\mathrm{O})$ attains $\mathbf{C}$. The extrusion of $\mathrm{ROH}$ from $\mathbf{C}$ affords $\mathbf{D}$, then forming lactone $\mathbf{E}$ which undergoes decarboxylation to generate azomethine ylide $\mathbf{F}$. Intermediate $\mathbf{G}$ is tautomeric isomer of $\mathbf{F}$ and sub- 
Table 1 Optimization of reaction conditions ${ }^{a}$<smiles>[R]OC/C=C/c1ccccc1</smiles><smiles>O=C(OC1CNC(C(=O)O)C1)c1ccccc1</smiles>

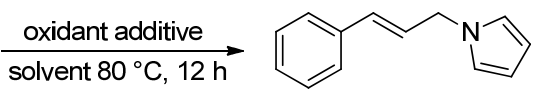

$3 \mathbf{a}$

\begin{tabular}{|c|c|c|c|c|}
\hline Entry & Oxidant & Solvent & Additive (equiv.) & Yield $^{b} / \%$ \\
\hline 1 & DDQ & Toluene & - & 27 \\
\hline $2^{c}$ & DDQ & Toluene & - & 11 \\
\hline 3 & $\mathrm{~T}^{+} \cdot \mathrm{BF}_{4}^{-}$ & Toluene & 一 & 19 \\
\hline 4 & DDQ & $\mathrm{PhCl}$ & - & 33 \\
\hline 5 & DDQ & DCE & - & 24 \\
\hline 6 & DDQ & $\mathrm{CCl}_{4}$ & - & 18 \\
\hline 7 & DDQ & THF & - & 20 \\
\hline 8 & DDQ & $\mathrm{CHCl}_{3}$ & - & 18 \\
\hline 9 & DDQ & EtOAc & - & 23 \\
\hline 10 & DDQ & $\mathrm{PhCl}$ & $\mathrm{InCl}_{3}(1.0)$ & 6 \\
\hline 11 & DDQ & $\mathrm{PhCl}$ & Phenol (1.0) & 38 \\
\hline 12 & DDQ & $\mathrm{PhCl}$ & TEMPO (1.0) & 27 \\
\hline 13 & DDQ & $\mathrm{PhCl}$ & Antioxidant $300(0.5)$ & 41 \\
\hline 14 & DDQ & $\mathrm{PhCl}$ & Antioxidant $300(1.0)$ & 57 \\
\hline 15 & DDQ & $\mathrm{PhCl}$ & Antioxidant 300 (2.0) & 33 \\
\hline $16^{e}$ & DDQ & $\mathrm{PhCl}$ & Antioxidant $300(1.0)$ & $71^{d}$ \\
\hline $17^{f}$ & DDQ & $\mathrm{PhCl}$ & Antioxidant $300(1.0)$ & $63^{d}$ \\
\hline $18^{g}$ & DDQ & $\mathrm{PhCl}$ & Antioxidant 300 (1.0) & $63^{d}$ \\
\hline $19^{h}$ & DDQ & $\mathrm{PhCl}$ & Antioxidant $300(1.0)$ & $60^{d}$ \\
\hline $20^{i}$ & DDQ & $\mathrm{PhCl}$ & Antioxidant $300(1.0)$ & $55^{d}$ \\
\hline
\end{tabular}

${ }^{a}$ Reaction conditions: 1 ( $0.2 \mathrm{mmol}, 1.0$ equiv. $), 2$ (0.4 mmol, 2.0 equiv.), oxidant ( $0.4 \mathrm{mmol}, 2.0$ equiv.), solvent $(3.0 \mathrm{~mL}), 80{ }^{\circ} \mathrm{C}, 12 \mathrm{~h} .{ }^{b}$ Determined by ${ }^{1} \mathrm{H}$ NMR analysis using dibromomethane as the internal standard. ${ }^{c} \mathbf{1}$ was oxidized by oxidant before adding $2 .{ }^{d}$ Isolated yields of reactions performed on 0.5 mmol scale. ${ }^{e} \mathrm{R}=$ ethyl. ${ }^{f} \mathrm{R}=$ isopropyl. ${ }^{g} \mathrm{R}=$ tert-butyl. ${ }^{h} 70{ }^{\circ} \mathrm{C} .{ }^{i} 90{ }^{\circ} \mathrm{C}$

sequent hydrogen migration of $\mathbf{G}$ provides H. Finally, elimination of $\mathbf{H}$ results in the formation of product $\mathbf{3}$.

\section{Conclusions}

In summary, a novel protocol has been demonstrated that tandem ipso-oxidation of allyl ether/decarboxylative aromatization generates $N$-alkyl pyrroles via oxocarbenium activation. This approach involves formation of $\mathrm{C}-\mathrm{N}$ bond and cleavage of $\mathrm{C}-\mathrm{O}$ bond. This method is complementary to the reported protocols for oxocarbenium activation. Moreover, this reaction allows convenient access to 1-cinnamyl-1H-pyrrole and its derivatives with good functional group tolerance and stimulates the design of new methods to synthesize $N$-alkyl pyrroles.

\section{Experimental section}

\subsection{General information}

All commercial reagents were used as received without further purification unless otherwise noted. Reaction tem-
Table 2 Substrate Scope ${ }^{a}$<smiles></smiles>

$\mathrm{DDQ}$

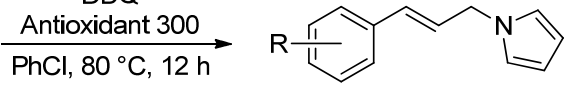

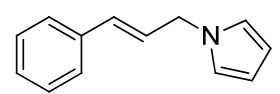

3a, $71 \%$

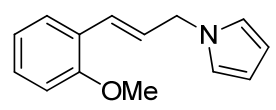

3c, $38 \%$<smiles>Cc1cccc(/C=C/Cn2cccc2)c1</smiles>

$\mathbf{3 e}, 31 \%$<smiles>Clc1ccc(/C=C/Cn2cccc2)cc1</smiles>

$3 g, 57 \%$<smiles>Clc1ccccc1/C=C/Cn1cccc1</smiles>

$3 i, 68 \%$<smiles>Fc1ccc(/C=C/Cn2cccc2)cc1</smiles>

$3 k, 57 \%$<smiles>C(=C/c1cccs1)\Cn1cccc1</smiles>

$3 m, 29 \%$<smiles>COc1ccc(/C=C/Cn2cccc2)cc1</smiles>

3b, $47 \%$<smiles>Cc1ccc(/C=C/Cn2cccc2)cc1</smiles>

3d, $56 \%$<smiles>Cc1ccccc1/C=C/Cn1cccc1</smiles>

3f, $69 \%$<smiles>Clc1cccc(/C=C/Cn2cccc2)c1</smiles>

$3 h, 55 \%$<smiles>Brc1ccc(/C=C/Cn2cccc2)cc1</smiles>

3j, $59 \%$<smiles>C(=C/c1ccco1)\Cn1cccc1</smiles>

3l, $41 \%$<smiles>FC(F)(F)c1ccc(/C=C/Cn2cccc2)cc1</smiles>

$3 n, 42 \%$

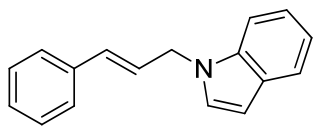

3o, $31 \%$

${ }^{a}$ Reaction conditions: 1 ( $0.5 \mathrm{mmol}, 1.0$ equiv.), 2 ( $1.0 \mathrm{mmol}, 2.0$ equiv.), DDQ (1.0 mmol, 2.0 equiv.), antioxidant 300 (0.5mmol, 1.0 equiv.), $\mathrm{PhCl}(10 \mathrm{~mL})$, $80{ }^{\circ} \mathrm{C}, 12 \mathrm{~h}$. Isolated yields.

peratures were reported as the temperatures of the bather surrounding the flasks or tubes. ${ }^{1} \mathrm{H}$ NMR and ${ }^{13} \mathrm{C}$ NMR spectra were recorded at a Bruke Avance-400 spectrometer (400 $\mathrm{MHz}$ and $100 \mathrm{MHz}$, respectively) using TMS as an internal standard. High-resolution mass spectra (HRMS) were carried out on Micromass GCTTM gas chromatograph-mass spectrometer. Flash chromatography was performed on silica gel $(300 \sim 400$ mesh) using mixtures of petroleum ether (b.p. $60 \sim 90{ }^{\circ} \mathrm{C}$ ) and ethyl acetate as elu- 


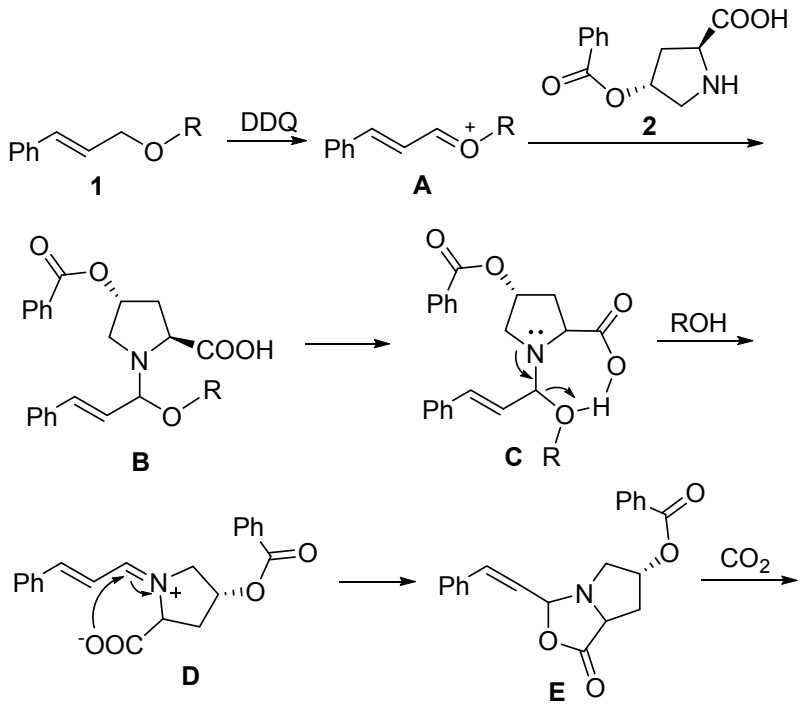

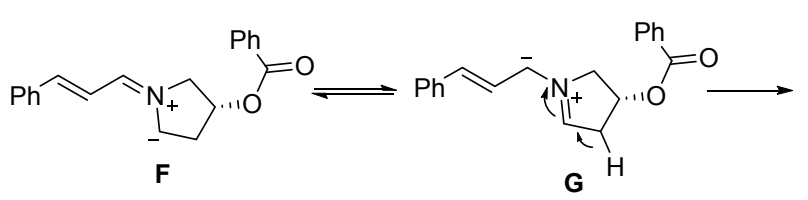<smiles>O=C(COC(=O)c1ccccc1)OC1C=CN(CC=Cc2ccccc2)C1</smiles>

Scheme 3 Proposed mechanism

ents.

4.2 Procedure for the synthesis of trans-4-benzoyloxy-L-proline 2

trans-4-Hydroxy- $L$-proline $(23.64 \mathrm{~g}, 180 \mathrm{mmol})$ was dissolved in $\mathrm{CF}_{3} \mathrm{CO}_{2} \mathrm{H}(100 \mathrm{~mL})$ by stirring at room temperature for $15 \mathrm{~min}$. The solution was cooled just below room temperature with an ice/water bath, removed from the bath, and benzoyl chloride $(41.8 \mathrm{~mL}, 360 \mathrm{mmol})$ was added in one portion (mildly exothermic). The solution was stirred at room temperature for $2 \mathrm{~h}$. Under cooling with an ice/water bath, $\mathrm{Et}_{2} \mathrm{O}(350 \mathrm{~mL})$ was added carefully. Stirring was discontinued, and the sticky precipitate was allowed to settle for $15 \mathrm{~min}$. The clear supernatant was decanted, and $i$-PrOH $(100 \mathrm{~mL})$ was added to the solid residue. Vigorous stirring at near reflux gave a fine white suspension. When it became homogeneous, it was cooled with an ice/water bath, and $\mathrm{Et}_{2} \mathrm{O}(200 \mathrm{~mL})$ was added whilst stirring. The resulting suspension was vacuum-filtered, washed with $\mathrm{Et}_{2} \mathrm{O}(50 \mathrm{~mL})$ and dried at room temperature for $16 \mathrm{~h}$ to give trans-4-benzoyloxy- $L$-proline hydrochloride as a fine white powder. All the hydrochloride salt was dissolved in $\mathrm{EtOH}(96 \mathrm{vol} \%, 100 \mathrm{~mL})$ by heating. The solution was cooled to approximately room temperature in an ice/water bath, removed from the bath, and propylene oxide $(20 \mathrm{~mL}, 286 \mathrm{mmol})$ was added. Stirring was discontinued and the solution left for crystallization at room temperature for $7 \mathrm{~h}$. The crystals were vacuum-filtered and dried at room temperature to give $\mathbf{2}$ as small colourless crystals. The product was recrystallized from boiling water $(160 \mathrm{~mL})$ to give $\mathbf{2}$ as colourless crystals.

\subsection{A typical procedure for the synthesis of the products 3}

An oven-dried $25 \mathrm{~mL}$ reaction flask equipped with a magnetic stirring bar was charged with $1(0.5 \mathrm{mmol}, 1.0$ equiv.), 2 (235.2 mg, $1.0 \mathrm{mmol}, 2.0$ equiv.), DDQ (227.0 $\mathrm{mg}, 1.0 \mathrm{mmol}, 2.0$ equiv.), Antioxidant 300 (179.3 mg, 0.5 mmol, 1.0 equiv.) and $\mathrm{PhCl}(10 \mathrm{~mL})$. The flask equipped with a reflux condenser was heated in an oil bath at $80{ }^{\circ} \mathrm{C}$ for $12 \mathrm{~h}$. The reaction was monitored by thin-layer chromatography (TLC). After cooling to room temperature, the resulting mixture was filtered through silica gel with EtOAc. The filtrate was removed in vacuo and the residue was purified by flash column chromatography (petroleum ether/ethyl acetate, $V: V=400: 1)$ to afford the desired products.

\subsection{Characterization data of the products}

1-Cinnamyl-1 $H$-pyrrole (3a): Yellow oil; yield $65 \mathrm{mg}$ (71\%). ${ }^{1} \mathrm{H}$ NMR $\left(400 \mathrm{MHz}, \mathrm{CDCl}_{3}\right) \delta: 7.39(\mathrm{~d}, J=6.9 \mathrm{~Hz}$, $2 \mathrm{H}), 7.34(\mathrm{t}, J=7.4 \mathrm{~Hz}, 2 \mathrm{H}), 7.28(\mathrm{~d}, J=7.1 \mathrm{~Hz}, 1 \mathrm{H}), 6.74$ (t, $J=2.1 \mathrm{~Hz}, 2 \mathrm{H}), 6.51(\mathrm{~d}, J=15.8 \mathrm{~Hz}, 1 \mathrm{H}), 6.36$ (dt, $J=$ $15.8,6.1 \mathrm{~Hz}, 1 \mathrm{H}), 6.22(\mathrm{t}, J=2.1 \mathrm{~Hz}, 2 \mathrm{H}), 4.67$ (dd, $J=$ $6.1,1.5 \mathrm{~Hz}, 2 \mathrm{H}) ;{ }^{13} \mathrm{C}$ NMR $\left(101 \mathrm{MHz}, \mathrm{CDCl}_{3}\right) \delta: 136.3$, 132.6, 128.6 (2C), 127.9, 126.6 (2C), 125.5, 120.7 (2C), 108.4 (2C), 51.6. HRMS (EI) calcd for $\mathrm{C}_{13} \mathrm{H}_{13} \mathrm{~N}$ : 183.1048 , found 183.1045 .

(E)-1-(3-(4-Methoxyphenyl)allyl)-1 $H$-pyrrole (3b): Yellow oil; yield $50 \mathrm{mg}(47 \%) .{ }^{1} \mathrm{H} \mathrm{NMR}\left(400 \mathrm{MHz}, \mathrm{CDCl}_{3}\right) \delta$ : $7.33(\mathrm{~d}, J=8.8 \mathrm{~Hz}, 2 \mathrm{H}), 6.87(\mathrm{~d}, J=8.7 \mathrm{~Hz}, 2 \mathrm{H}), 6.74(\mathrm{t}$, $J=2.2 \mathrm{~Hz}, 2 \mathrm{H}), 6.47(\mathrm{~d}, J=15.8 \mathrm{~Hz}, 1 \mathrm{H}), 6.26 \sim 6.19(\mathrm{~m}$, $3 \mathrm{H}), 4.65(\mathrm{~d}, J=6.3 \mathrm{~Hz}, 2 \mathrm{H}), 3.83(\mathrm{~s}, 3 \mathrm{H}) ;{ }^{13} \mathrm{C}$ NMR $(101$ $\left.\mathrm{MHz}, \mathrm{CDCl}_{3}\right) \delta: 159.5,132.1,129.1,127.8$ (2C), 123.2, 120.6 (2C), 114.0 (2C), 108.3 (2C), 55.3, 51.7. HRMS (EI) calcd for $\mathrm{C}_{14} \mathrm{H}_{15} \mathrm{NO}$ : 213.1154, found 213.1156.

(E)-1-(3-(2-Methoxyphenyl)allyl)-1 $H$-pyrrole (3c): Yellow oil; yield $41 \mathrm{mg}(38 \%) .{ }^{1} \mathrm{H}$ NMR $\left(400 \mathrm{MHz}, \mathrm{CDCl}_{3}\right) \delta$ : 7.43 (dd, $J=7.7,1.7 \mathrm{~Hz}, 1 \mathrm{H}), 7.29 \sim 7.22(\mathrm{~m}, 1 \mathrm{H}), 6.98 \sim$ $6.86(\mathrm{~m}, 3 \mathrm{H}), 6.75(\mathrm{t}, J=2.1 \mathrm{~Hz}, 2 \mathrm{H}), 6.38(\mathrm{dt}, J=15.9$, $6.5 \mathrm{~Hz}, 1 \mathrm{H}), 6.20(\mathrm{t}, J=2.1 \mathrm{~Hz}, 2 \mathrm{H}), 4.68(\mathrm{dd}, J=6.5,1.5$ $\mathrm{Hz}, 2 \mathrm{H}), 3.87(\mathrm{~s}, 3 \mathrm{H}) ;{ }^{13} \mathrm{C}$ NMR $\left(101 \mathrm{MHz}, \mathrm{CDCl}_{3}\right) \delta$ : $156.8,129.0,127.9,127.1,126.0,125.3,120.7,120.6$ (2C), 110.9, 108.2 (2C), 55.5, 52.2. HRMS (EI) calcd for $\mathrm{C}_{14} \mathrm{H}_{15} \mathrm{NO}: 213.1154$, found 213.1150 .

(E)-1-(3-(p-Tolyl)allyl)-1H-pyrrole (3d): Yellow oil; yield $55 \mathrm{mg}(56 \%) .{ }^{1} \mathrm{H}$ NMR $\left(400 \mathrm{MHz}, \mathrm{CDCl}_{3}\right) \delta: 7.30(\mathrm{~d}$, $J=8.1 \mathrm{~Hz}, 2 \mathrm{H}), 7.16(\mathrm{~d}, J=7.9 \mathrm{~Hz}, 2 \mathrm{H}), 6.75(\mathrm{t}, J=2.1$ $\mathrm{Hz}, 2 \mathrm{H}), 6.51(\mathrm{~d}, J=15.8 \mathrm{~Hz}, 1 \mathrm{H}), 6.32(\mathrm{dt}, J=15.8,6.2$ $\mathrm{Hz}, 1 \mathrm{H}), 6.23(\mathrm{t}, J=2.1 \mathrm{~Hz}, 2 \mathrm{H}), 4.67(\mathrm{dd}, J=6.2,1.4 \mathrm{~Hz}$, 2H), $2.38(\mathrm{~s}, 3 \mathrm{H}) ;{ }^{13} \mathrm{C}$ NMR $\left(101 \mathrm{MHz}, \mathrm{CDCl}_{3}\right) \delta: 137.8$, 133.5, 132.5, 129.4 (2C), 126.5 (2C), 124.5, 120.7 (2C), 108.4 (2C), 51.7, 21.3. HRMS (EI) calcd for $\mathrm{C}_{14} \mathrm{H}_{15} \mathrm{~N}$ : 197.1204, found 197.1201.

(E)-1-(3-( $m$-Tolyl)allyl)-1 $H$-pyrrole (3e): Yellow oil; yield $31 \mathrm{mg}(31 \%)$. ${ }^{1} \mathrm{H}$ NMR $\left(400 \mathrm{MHz}, \mathrm{CDCl}_{3}\right) \delta: 7.25 \sim$ 7.19 (m, 3H), 7.09 (d, $J=7.3 \mathrm{~Hz}, 1 \mathrm{H}), 6.73$ (t, $J=2.1 \mathrm{~Hz}$, 
2H), $6.48(\mathrm{~d}, J=15.8 \mathrm{~Hz}, 1 \mathrm{H}), 6.34(\mathrm{dt}, J=15.7,6.1 \mathrm{~Hz}$, $1 \mathrm{H}), 6.21(\mathrm{t}, J=2.1 \mathrm{~Hz}, 2 \mathrm{H}), 4.66(\mathrm{dd}, J=6.1,1.4 \mathrm{~Hz}$, 2H), $2.36(\mathrm{~s}, 3 \mathrm{H}) ;{ }^{13} \mathrm{C}$ NMR $\left(101 \mathrm{MHz}, \mathrm{CDCl}_{3}\right) \delta: 138.2$, $136.2,132.7,128.7,128.5,127.3,125.3,123.7,120.7$ (2C), 108.4 (2C), 51.6, 21.4. HRMS (EI) calcd for $\mathrm{C}_{14} \mathrm{H}_{15} \mathrm{~N}$ : 197.1204, found 197.1201.

(E)-1-(3-(o-Tolyl)allyl)-1H-pyrrole (3f): Yellow oil; yield $68 \mathrm{mg}(69 \%) .{ }^{1} \mathrm{H}$ NMR $\left(400 \mathrm{MHz}, \mathrm{CDCl}_{3}\right) \delta: 7.49 \sim$ $7.45(\mathrm{~m}, 1 \mathrm{H}), 7.24 \sim 7.20(\mathrm{~m}, 3 \mathrm{H}), 6.78(\mathrm{t}, J=2.1 \mathrm{~Hz}, 2 \mathrm{H})$, $6.72(\mathrm{~d}, J=15.9 \mathrm{~Hz}, 1 \mathrm{H}), 6.31 \sim 6.23(\mathrm{~m}, 3 \mathrm{H}), 4.72(\mathrm{dd}$, $J=6.2,1.6 \mathrm{~Hz}, 2 \mathrm{H}), 2.39$ (s, 3H); ${ }^{13} \mathrm{C}$ NMR $(101 \mathrm{MHz}$, $\left.\mathrm{CDCl}_{3}\right) \delta: 135.6,135.5,130.6,130.4,127.9,126.9,126.2$, 125.9, 120.7 (2C), 108.4 (2C), 51.9, 19.8. HRMS (EI) calcd for $\mathrm{C}_{14} \mathrm{H}_{15} \mathrm{~N}$ : 197.1204, found 197.1209.

(E)-1-(3-(4-Chlorophenyl)allyl)-1H-pyrrole (3g): Yellow oil; yield $62 \mathrm{mg}(57 \%) .{ }^{1} \mathrm{H} \mathrm{NMR}\left(400 \mathrm{MHz}, \mathrm{CDCl}_{3}\right) \delta$ : $7.32(\mathrm{~s}, 4 \mathrm{H}), 6.75(\mathrm{t}, J=2.0 \mathrm{~Hz}, 2 \mathrm{H}), 6.45(\mathrm{~d}, J=15.8 \mathrm{~Hz}$, $1 \mathrm{H}), 6.34(\mathrm{dt}, J=15.8,5.9 \mathrm{~Hz}, 1 \mathrm{H}), 6.25(\mathrm{t}, J=2.0 \mathrm{~Hz}$, $2 \mathrm{H}), 4.68$ (dd, $J=5.9,1.3 \mathrm{~Hz}, 2 \mathrm{H}) ;{ }^{13} \mathrm{C} \mathrm{NMR}(101 \mathrm{MHz}$, $\left.\mathrm{CDCl}_{3}\right) \delta: 134.8,133.6,131.2,128.8(2 \mathrm{C}), 127.8(2 \mathrm{C})$, 126.4, 120.7 (2C), 108.6 (2C), 51.5. HRMS (EI) calcd for $\mathrm{C}_{13} \mathrm{H}_{12} \mathrm{ClN}$ : 217.0658, found 217.0655 .

(E)-1-(3-(3-Chlorophenyl)allyl)-1H-pyrrole (3h): Yellow oil; yield $60 \mathrm{mg}(55 \%)$. ${ }^{1} \mathrm{H} \mathrm{NMR}\left(400 \mathrm{MHz}, \mathrm{CDCl}_{3}\right) \delta$ : $7.38(\mathrm{~s}, 1 \mathrm{H}), 7.29 \sim 7.23(\mathrm{~m}, 3 \mathrm{H}), 6.74(\mathrm{t}, J=2.0 \mathrm{~Hz}, 2 \mathrm{H})$, $6.42(\mathrm{~d}, J=16.8 \mathrm{~Hz}, 1 \mathrm{H}), 6.37$ (dt, $J=16.7,5.2 \mathrm{~Hz}, 1 \mathrm{H})$, $6.25(\mathrm{t}, J=2.0 \mathrm{~Hz}, 2 \mathrm{H}), 4.68(\mathrm{~d}, J=5.0 \mathrm{~Hz}, 2 \mathrm{H}) ;{ }^{13} \mathrm{C} \mathrm{NMR}$ $\left(101 \mathrm{MHz}, \mathrm{CDCl}_{3}\right) \delta: 138.2,134.6,131.0,129.9,127.9$, 127.3, 126.5, 124.7, 120.7 (2C), 108.6 (2C), 51.4. HRMS (EI) calcd for $\mathrm{C}_{13} \mathrm{H}_{12} \mathrm{ClN}$ : 217.0658, found 217.0662 .

(E)-1-(3-(2-Chlorophenyl)allyl)-1 $H$-pyrrole (3i): Yellow oil; yield $74 \mathrm{mg}(68 \%) .{ }^{1} \mathrm{H}$ NMR $\left(400 \mathrm{MHz}, \mathrm{CDCl}_{3}\right) \delta$ : 7.55 (dd, $J=7.2,2.4 \mathrm{~Hz}, 1 \mathrm{H}), 7.42$ (dd, $J=7.3,2.0 \mathrm{~Hz}$, 1H), $7.28 \sim 7.23(\mathrm{~m}, 2 \mathrm{H}), 6.99(\mathrm{~d}, J=15.8 \mathrm{~Hz}, 1 \mathrm{H}), 6.79$ $(\mathrm{t}, J=2.1 \mathrm{~Hz}, 2 \mathrm{H}), 6.36(\mathrm{dt}, J=15.8,6.3 \mathrm{~Hz}, 1 \mathrm{H}), 6.28(\mathrm{t}$, $J=2.2 \mathrm{~Hz}, 2 \mathrm{H}), 4.73(\mathrm{dd}, J=6.3,1.7 \mathrm{~Hz}, 2 \mathrm{H}) ;{ }^{13} \mathrm{C} \mathrm{NMR}$ $\left(101 \mathrm{MHz}, \mathrm{CDCl}_{3}\right) \delta: 134.5,133.2,129.8,129.0,129.0$, 128.5, 127.1, 127.0, 120.7 (2C), 108.6 (2C), 51.7. HRMS (EI) calcd for $\mathrm{C}_{13} \mathrm{H}_{12} \mathrm{CIN}$ : 217.0658, found 217.0660.

(E)-1-(3-(4-Bromophenyl)allyl)-1 $H$-pyrrole (3j): Yellow oil; yield $77 \mathrm{mg}(59 \%) .{ }^{1} \mathrm{H}$ NMR $\left(400 \mathrm{MHz}, \mathrm{CDCl}_{3}\right) \delta$ : 7.39 (d, $J=8.5 \mathrm{~Hz}, 2 \mathrm{H}), 7.16(\mathrm{~d}, J=8.5 \mathrm{~Hz}, 2 \mathrm{H}), 6.67$ (t, $J=2.1 \mathrm{~Hz}, 2 \mathrm{H}), 6.34(\mathrm{~d}, J=15.9 \mathrm{~Hz}, 1 \mathrm{H}), 6.26(\mathrm{dt}, J=$ $15.8,5.6 \mathrm{~Hz}, 1 \mathrm{H}), 6.18(\mathrm{t}, J=2.1 \mathrm{~Hz}, 2 \mathrm{H}), 4.58(\mathrm{~d}, J=5.6$ $\mathrm{Hz}, 2 \mathrm{H}) ;{ }^{13} \mathrm{C}$ NMR $\left(101 \mathrm{MHz}, \mathrm{CDCl}_{3}\right) \delta: 135.3,131.8$ (2C), 131.2, 128.1 (2C), 126.6, 121.8, 120.8 (2C), 108.6 (2C), 51.5. HRMS (EI) calcd for $\mathrm{C}_{13} \mathrm{H}_{12} \mathrm{BrN}$ : 261.0153, found 261.0156

(E)-1-(3-(4-Fluorophenyl)allyl)-1H-pyrrole (3k): Yellow oil; yield $57 \mathrm{mg}(57 \%) .{ }^{1} \mathrm{H}$ NMR $\left(400 \mathrm{MHz}, \mathrm{CDCl}_{3}\right) \delta$ : 7.35 (dd, $J=8.6,5.5 \mathrm{~Hz}, 2 \mathrm{H}), 7.02$ (t, $J=8.7 \mathrm{~Hz}, 2 \mathrm{H}), 6.73$ $(\mathrm{t}, J=2.1 \mathrm{~Hz}, 2 \mathrm{H}), 6.46(\mathrm{~d}, J=15.8 \mathrm{~Hz}, 1 \mathrm{H}), 6.27$ (dt, $J=$ $15.9,6.1 \mathrm{~Hz}, 1 \mathrm{H}), 6.22(\mathrm{t}, J=2.1 \mathrm{~Hz}, 2 \mathrm{H}), 4.66(\mathrm{dd}, J=$ 6.1, 1.6 Hz, 2H); ${ }^{13} \mathrm{C}$ NMR (101 MHz, $\left.\mathrm{CDCl}_{3}\right) \delta: 162.5(\mathrm{~d}$, $J=247.3 \mathrm{~Hz}), 132.5(\mathrm{~d}, J=3.4 \mathrm{~Hz}), 131.3,128.1(\mathrm{~d}, J=$ $8.0 \mathrm{~Hz}, 2 \mathrm{C}), 125.4$ (d, $J=2.2 \mathrm{~Hz}), 120.7$ (2C), 115.6 (d, $J=21.6 \mathrm{~Hz}, 2 \mathrm{C}$ ), 108.5 (2C), 51.5. HRMS (EI) calcd for
$\mathrm{C}_{13} \mathrm{H}_{12} \mathrm{FN}$ : 201.0954, found 201.0950.

(E)-1-(3-(Furan-2-yl)allyl)-1H-pyrrole (31): Yellow oil; yield $35 \mathrm{mg}(41 \%) .{ }^{1} \mathrm{H}$ NMR $\left(400 \mathrm{MHz}, \mathrm{CDCl}_{3}\right) \delta: 7.34(\mathrm{~d}$, $J=1.7 \mathrm{~Hz}, 1 \mathrm{H}), 6.71(\mathrm{t}, J=2.1 \mathrm{~Hz}, 2 \mathrm{H}), 6.37(\mathrm{dd}, J=3.3$, $1.9 \mathrm{~Hz}, 1 \mathrm{H}), 6.29(\mathrm{dt}, J=15.8,5.5 \mathrm{~Hz}, 1 \mathrm{H}), 6.23(\mathrm{~d}, J=2.5$ $\mathrm{Hz}, 2 \mathrm{H}), 6.19$ (t, $J=2.1 \mathrm{~Hz}, 2 \mathrm{H}), 4.64$ (d, $J=5.4 \mathrm{~Hz}, 2 \mathrm{H})$. ${ }^{13} \mathrm{C}$ NMR $\left(101 \mathrm{MHz} \mathrm{CDCl}_{3}\right) \delta: 152.0,142.2,124.3,120.8$ (2C), 120.5, 111.3, 108.4, 108.4 (2C), 51.2. HRMS (EI) calcd for $\mathrm{C}_{11} \mathrm{H}_{11} \mathrm{NO}$ : 173.0841, found 173.0844.

(E)-1-(3-(Thiophen-2-yl)allyl)-1 $H$-pyrrole (3m): Yellow oil; yield $27 \mathrm{mg}(29 \%)$. ${ }^{1} \mathrm{H}$ NMR (400 $\left.\mathrm{MHz}, \mathrm{CDCl}_{3}\right) \delta$ : $7.20 \sim 7.16(\mathrm{~m}, 1 \mathrm{H}), 7.00 \sim 6.96(\mathrm{~m}, 2 \mathrm{H}), 6.73(\mathrm{t}, J=2.1$ $\mathrm{Hz}, 2 \mathrm{H}), 6.60(\mathrm{~d}, J=15.6 \mathrm{~Hz}, 1 \mathrm{H}), 6.22(\mathrm{t}, J=2.2 \mathrm{~Hz}, 2 \mathrm{H})$, $6.22 \sim 6.13(\mathrm{~m}, 1 \mathrm{H}), 4.63(\mathrm{dd}, J=6.1,1.6 \mathrm{~Hz}, 2 \mathrm{H}) ;{ }^{13} \mathrm{C}$ NMR (101 MHz, $\left.\mathrm{CDCl}_{3}\right) \delta$ : 141.3, 127.4, 126.2, 125.6, 125.1, 124.7, 120.7 (2C), 108.4 (2C), 51.3. HRMS (EI) calcd for $\mathrm{C}_{11} \mathrm{H}_{11} \mathrm{NS}$ : 189.0612 , found 189.0605 .

(E)-1-(3-(4-(Trifluoromethyl)phenyl)allyl)-1 $H$-pyrrole (3n): Yellow oil; yield $53 \mathrm{mg}(42 \%) .{ }^{1} \mathrm{H}$ NMR $(400 \mathrm{MHz}$, $\left.\mathrm{CDCl}_{3}\right) \delta: 7.60(\mathrm{~d}, J=8.2 \mathrm{~Hz}, 2 \mathrm{H}), 7.48(\mathrm{~d}, J=8.1 \mathrm{~Hz}$, $2 \mathrm{H}), 6.75(\mathrm{t}, J=2.1 \mathrm{~Hz}, 2 \mathrm{H}), 6.54 \sim 6.41(\mathrm{~m}, 2 \mathrm{H}), 6.26(\mathrm{t}$, $J=2.1 \mathrm{~Hz}, 2 \mathrm{H}), 4.71(\mathrm{~d}, J=4.4 \mathrm{~Hz}, 2 \mathrm{H}) ;{ }^{13} \mathrm{C}$ NMR $(101$ $\left.\mathrm{MHz}, \mathrm{CDCl}_{3}\right) \delta: 139.8,130.9,129.7(\mathrm{q}, J=32.4 \mathrm{~Hz})$, $128.5,126.7$ (2C), 125.6 (q, $J=3.8 \mathrm{~Hz}, 2 \mathrm{C}), 124.2$ (q, $J=$ $271.8 \mathrm{~Hz}), 120.8$ (2C), 108.7 (2C), 51.4. HRMS (EI) calcd for $\mathrm{C}_{14} \mathrm{H}_{12} \mathrm{~F}_{3} \mathrm{~N}$ : 251.0922, found 251.0927 .

1-Cinnamyl-1 $H$-indole (3o) Yellow oil; yield $36 \mathrm{mg}$ (31\%). ${ }^{1} \mathrm{H}$ NMR $\left(400 \mathrm{MHz}, \mathrm{CDCl}_{3}\right) \delta: 7.64$ (d, $J=7.8 \mathrm{~Hz}$, $1 \mathrm{H}), 7.36(\mathrm{~d}, J=8.2 \mathrm{~Hz}, 1 \mathrm{H}), 7.33 \sim 7.28(\mathrm{~m}, 3 \mathrm{H}), 7.26 \sim$ $7.17(\mathrm{~m}, 3 \mathrm{H}), 7.14 \sim 7.08(\mathrm{~m}, 2 \mathrm{H}), 6.53(\mathrm{~d}, J=3.2 \mathrm{~Hz}$, $1 \mathrm{H}), 6.46(\mathrm{~d}, J=15.8 \mathrm{~Hz}, 1 \mathrm{H}), 6.31(\mathrm{dt}, J=15.9,5.8 \mathrm{~Hz}$, $1 \mathrm{H}), 4.85(\mathrm{dd}, J=5.7,1.5 \mathrm{~Hz}, 2 \mathrm{H}) ;{ }^{13} \mathrm{C} \mathrm{NMR}(101 \mathrm{MHz}$, $\left.\mathrm{CDCl}_{3}\right) \delta: 136.3,136.2,132.4,128.8,128.6$ (2C), 127.9, $127.8,126.5$ (2C), 124.9, 121.7, 121.0, 119.5, 109.7, 101.6, 48.4. HRMS (EI) calcd for $\mathrm{C}_{17} \mathrm{H}_{15} \mathrm{~N}$ : 233.1204, found 233.1202 .

Supporting Information ${ }^{1} \mathrm{H}$ NMR and ${ }^{13} \mathrm{C}$ NMR spectra of compounds $\mathbf{3 a} \sim \mathbf{3 o}$. The Supporting Information is available free of charge via the Internet at http://siocjournal.cn/.

\section{References}

[1] Curran, D.; Grimshaw, J.; Perera, S. D. Chem. Soc. Rev. 1991, 20, 391.

[2] (a) Azizian, J.; Karimi, A. R.; Kazemizadeh, Z.; Mohammadi, A. A.; Mohammadizadeh, M. R. J. Org. Chem. 2005, 70, 1471. (b) Meshram, H. M.; Prasad, B. R. V.; Aravind Kumar, D. Tetrahedron Lett. 2010, 51, 3477.

(c) Deb, I.; Coiro, D. J.; Seidel, D. Chem. Commun. 2011, 47, 6473. (d) Mao, H.; Wang, S.; Yu, P.; Lv, H.; Xu, R.; Pan, Y. J. Org. Chem. 2011, 76, 1167.

(e) Zou, Z.; Deng, Z.; Yu, X.; Zhang, M.; Zhao, S.; Luo, T.; Yin, X.; Xu, H.; Wang, W. Sci. China Chem. 2012, 55, 43.

(f) Satish, G.; Reddy, K. H. V.; Ramesh, K.; Kumar, B. S. P. A.; Nageswar, Y. V. D. Tetrahedron Lett. 2014, 55, 2596.

[3] (a) Miller, A. J. M.; Kaminsky, W.; Goldberg, K. I. Organometallics 2014, 33, 1245.

(b) Song, Z.-Z.; Gong, J.-L.; Zhang, M.; Wu, X.-F. Asian J. Org. 
Chem. 2012, 1, 214.

(c) Shen, Z.; Dai, J.; Xiong, J.; He, X.; Mo, W.; Hu, B.; Sun, N.; Hu, X. Adv. Synth. Catal. 2011, 353, 3031.

[4] (a) Zhang, Y.; Li, C.-J. Angew. Chem., Int. Ed. 2006, 45, 1949.

(b) Zhang, Y.; Li, C.-J. J. Am. Chem. Soc. 2006, 128, 4242.

(c) Tu, W.; Liu, L.; Floreancig, P. E. Angew. Chem., Int. Ed. 2008, 47, 4184.

(d) Tu, W.; Floreancig, P. E. Angew. Chem., Int. Ed. 2009, 48, 4567.

(e) Liu, L.; Floreancig, P. E. Angew. Chem., Int. Ed. 2010, 49, 5894.

(f) Richter, H.; Rohlmann, R.; García Mancheño, O. Chem.-Eur. J. 2011, 17, 11622.

(g) Muramatsu, W.; Nakano, K.; Li, C.-J. Org. Lett. 2013, 15, 3650.

(h) Meng, Z.; Sun, S.; Yuan, H.; Lou, H.; Liu, L. Angew. Chem. Int. Ed. 2014, 53, 543.

(i) Chen, W.; Xie, Z.; Zheng, H.; Lou, H.; Liu, L. Org. Lett. 2014, $16,5988$.

(j) Yu, B.; Jiang, T.; Li, J.; Su, Y.; Pan, X.; She, X. Org. Lett. 2009, 11,3442 .

(k) Liu, L.; Floreancig, P. E. Org. Lett. 2010, 12, 4686.

(1) Liu, X.; Sun, B.; Xie, Z.; Qin, X.; Liu, L.; Lou, H. J. Org. Chem. 2013, 78, 3104 .

(m) Sun, K.; Wang, X.; Li, G.; Zhu, Z.; Jiang, Y.; Xiao, B. Chem. Commun. 2014, 50, 12880. (n) Pandit, R. P.; Lee, Y. R. Adv. Synth. Catal. 2014, 356, 3171

[5] (a) Reisman, S. E.; Doyle, A. G.; Jacobsen, E. N. J. Am. Chem. Soc. 2008, 130, 7198 .

(b) McQuaid, K. M.; Sames, D. J. Am. Chem. Soc. 2009, 131, 402. (c) Maity, P.; Srinivas, H. D.; Watson, M. P. J. Am. Chem. Soc. 2011, 133, 17142.

(d) Chen, Y.; Chen, M.; Liu, Y. Angew. Chem. 2012, 124, 6599.

(e) Rueping, M.; Volla, C. M. R.; Atodiresei, I. Org. Lett. 2012, 14, 4642.

(f) Lu, C.; Su, X.; Floreancig, P. E. J. Org. Chem. 2013, 78, 9366. (g) Srinivas, H. D.; Maity, P.; Yap, G. P. A.; Watson, M. P. J. Org. Chem. 2015, 80, 4003.

(h) Kong, A.; Andreansky, E. S.; Blakey, S. B. J. Org. Chem. 2017, $82,4477$.

(i) Fan, W.-T.; Li, N.-K.; Xu, L.; Qiao, C.; Wang, X.-W. Org. Lett. 2017, 19, 6626.

(j) Lee, S.; Kaib, P. S. J.; List, B. J. Am. Chem. Soc. 2017, 139, 2156.

[6] (a) Zhang, S.-Y.; Zhang, F.-M.; Tu, Y.-Q. Chem. Soc. Rev. 2011, 40, 1937.

(b) Morales-Rivera, C. A.; Floreancig, P. E.; Liu, P. J. Am. Chem. Soc. 2017, 139, 17935.

(Li, L.; Fan, Y.) 